\title{
POWER OPTIMIZED SPACE-TIME CODE FOR LAYER CODED MULTIMEDIA OVER WIRELESS CHANNELS
}

\author{
H. Zheng and K. J. R. Liu \\ Department of Electrical Engineering and Institute for Systems Research \\ University of Maryland,College Park, MD 20742 \\ Tel: 301-405-6619, e-mail: haitaoz, kjrliu@eng.umd.edu
}

\begin{abstract}
A power optimized space-time code is presented for reliable delivery of layer coded multimedia data through wireless channels. Appropriate power control further improves the performance of space time coding. By allocating transmitted power according to the layer perceptual importance, unequal error protection is achieved. Numerical example shows that the proposed algorithm achieves 6dB PSNR improvement compared to that with equal power distribution in low SNR range.
\end{abstract}

\section{INTRODUCTION}

The effect of multipath fading in wireless channel can be substantially reduced through the use of diversity techniques. By using multiple antennas at the receiver, multiple copies of the transmitted data are received so that the outrage probability can be reduced. This is referred to as receive diversity. However, receive diversity can be expensive or impractical when the receiver is a small hand-held unit. In such case, transmit diversity which uses multiple transmit antennas at the transmitter becomes more effective. Many researchers have studied transmit diversity. Linear transform is used at the transmitter to spread the information across the antennas[1]. The transmit diversity gain with ideal MLSE and an arbitrary number of antennas is compared with receive diversity in [2]. A Linear antenna precoding proposed in [3] can transform a nonselective Rayleigh fading channel into a non-fading, simple, marginally Gaussian noise channel, without intersymbol interference, given a sufficient number of transmit antennas.

Recently, the space-time code which is a joint design of coding, modulation, transmit and receive diversity, has been developed for high data rate wireless communications[4]. Input data is encoded by a channel code and then splitted into multiple streams, transmitted simultaneously. At each receive antenna, the received signal is a superposition of the transmitted

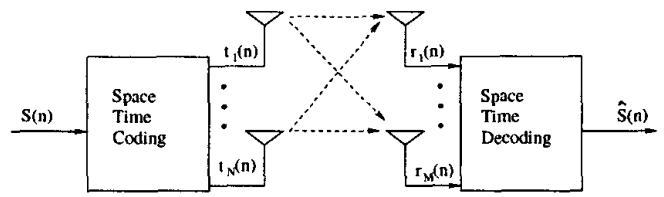

Figure 1: Space-time coding

signals perturbed by noise. The number of the streams and the number of transmit antenna are fixed to the same to avoid sacrifice in transmission rate. A spacetime block coded is developed to reduce the decoding complexity at the receiver.

Multimedia wireless service requires the transmission of multimedia data over wireless channels. The input data is represented in terms of a number of layers, each resulting in a distinct data stream, which represents different perceptually relevant components of source material. The different layers may have distinctly different tolerances to channel errors and the corresponding data streams can then be handled differently by the transmitter. Unequal error protection shows significant performance improvement over equal error protection[7, 8]. Current diversity techniques are all developed for data transmission where the input data are of equal importance, therefore providing only equal error protection. In this paper, we intend to realize unequal error protection by combining power control and transmit diversity techniques. We propose a power optimized space-time code which assigns the transmitted power at each antenna according to the layer to be transmitted.

\section{SPACE-TIME CODING}

Space-time coding with $N$ transmit antennas and $M$ receiver antennas are shown in Figure 1. At time $n$, the input data $s(n)$ is encoded as $N$ code symbols $t_{1}(n), t_{2}(n), \ldots ., t_{N}(n)$. Assuming that $t_{i}(n)$ is normal- 


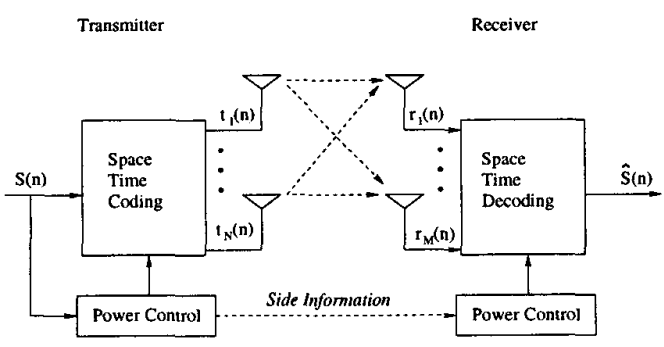

Figure 2: Power optimized space-time coding

ized by the average power $E_{s}$, the signal at each receiver antenna is a noisy, linear superposition of the distorted $N$ transmitted signals,

$$
r_{j}(n)=\sqrt{E_{s}} \sum_{i=1}^{N} H_{i, j}(n) t_{i}(n)+w_{j}(n), j=1 \ldots M,
$$

where $H_{i, j}(n)$ is the channel gain at time $n$, corresponding to the $i$-th transmit antenna and the $j$-th receive antenna, and $w_{j}(n)$ denotes the additive complex white Gaussian noise. It is assumed that $H_{i, j}(n)$ and $H_{p, q}(n)$ are independent for $i \neq p$ and $j \neq q$. The matrix form of (1) is given by

$$
R(n)=\sqrt{E_{s}} H(n) T(n)+W(n)
$$

with received signal vector $R(n)=\left[r_{1}(n), r_{2}(n)\right.$, $\left.\ldots, r_{M}(n)\right]^{T}$, transmitted signal vector $T(n)=$ $\left[t_{1}(n), t_{2}(n), \ldots, t_{N}(n)\right]^{T}$ and noise vector $W(n)=$ $\left[w_{1}(n), w_{2}(n), \ldots, w_{M}(n)\right]^{T} . H(n)$ is a $M \times N$ matrix, representing the channel gain from the $N$ transmit antennas to the $M$ receive antennas. In [4], the average pairwise error probability has the form of

$$
P e \leq\left(\prod_{i=1}^{r} \lambda_{i}\right)^{-M} \cdot\left(\frac{E_{s}}{4 N_{0}}\right)^{-r M}
$$

where $\left(\lambda_{1}, \lambda_{2}, \ldots, \lambda_{r}\right)$ and $r$ are the nonzero eigenvalues and rank of the matrix $\sum_{n=1}^{L}\left(T_{n}-\hat{T}_{n}\right)\left(T_{n}-\hat{T}_{n}\right)^{H}$, respectively.

\section{POWER OPTIMIZATION TO MINIMIZE DISTORTION}

From (3), the error probability is a monotonic function of transmitted power. Therefore, unequal error protection can be achieved by varying the transmitted power during each layer transmission, as shown in Figure 2. The goal of optimization is to minimize the overall distortion under the power constraint. The distortion is defined as the mean square error between the transmitted and received data. Assuming the orthogonality among the layers, the distortion can be approximated as the sum of the distortion at each layer. Similarly to [8], such optimization is formulated as

$$
\begin{aligned}
\text { Min } \quad D_{c} \approx \sum_{k=1}^{K} W_{k} \operatorname{Pe}\left(E_{k}\right), \\
\text { subject to } \quad \sum_{k=1}^{K} L_{k} E_{k} \leq E_{T} \sum_{k=1}^{K} L_{k},
\end{aligned}
$$

where $W_{k}$ denotes the average distortion caused by a bit error at layer $k, \operatorname{Pe}\left(E_{k}\right)$ is the bit error probability if transmitted using power $E_{k}$, and $L_{k}$ is the number of symbol of layer $k$ to be transmitted. If using space-time code, and transmitting the layers consecutively,

$$
P e\left(E_{k}\right) \propto \phi_{k} \cdot\left(\frac{E_{k}}{4 N_{0}}\right)^{-r_{k} M}
$$

where $\phi_{k}$ is the $\min \left\{\left(\prod_{i=1}^{r} \lambda_{i}\right)^{-M}\right\}$ of all the possible symbol sequences of layer $k$, and $r_{k}$ is the minimum of all the $r$ of subband $k$. If the same space-time code is applied, it is fair to assume that all layers have the same $\phi$ and $r$. Therefore, (4) is equivalent to minimize

$$
\sum_{k=1}^{K} W_{k}\left(\frac{E_{k}}{4 N_{0}}\right)^{-r M}
$$

Using LaGrange multiplier, we get

$$
E_{k}=E_{T} \frac{\sum_{i=1}^{K} L_{i} \cdot\left(W_{k}\right)^{\frac{1}{r M}}}{\sum_{i=1}^{K} L_{i}\left(W_{i}\right)^{\frac{1}{r M}}} .
$$

\section{POWER OPTIMIZATION FOR GIVEN QOS REQUIREMENT}

Typical multimedia data stream include video, image, audio, speech or the integrated service which contains two or more different data streams. Those streams have different quality of service(QoS) in terms of bit error rate(BER), as well as different data rate. For some applications, the customers can select the QoS of the layers and such information is available at the transmitter before the transmission. Therefore, the goal becomes to achieve the predefined QoS requirement while using the minimum amount of channel resources. Such consideration would help to increase the number of users that the system can support. We define the QoS of layer $k$ in terms of BER of layer $k$ as $P e_{k}$. Due to the difficulty in computing the $\phi_{k}$ of the bit error probability (5), we define a factor $P$ where $P e_{k}=Q_{k} \cdot P, \quad k=1 \ldots K$. We consider the problem of achieving the BER distribution of $P e_{k}=Q_{k} \cdot P$ and minimizing $P$. Here $Q_{k}$ 
can be deemed as a measurement of importance, large $Q_{k}$ leads to low importance. The goal is then

$$
\begin{aligned}
\text { Min } & P \\
\text { where } & P e_{k}=Q_{k} \cdot P, \quad k=1 . . K, \\
\text { subject to } & \sum_{k=1}^{K} L_{k} E_{k} \leq E_{T} \sum_{k=1}^{K} L_{k},
\end{aligned}
$$

where $L_{k}$ is the data rate of stream $k$.

The requirement $P e_{k}=Q_{k} \cdot P, \quad k=1 \ldots K$ leads to

$$
\frac{\left(\frac{E_{k}}{4 N_{0}}\right)^{-r M}}{Q_{k}}=\frac{\left(\frac{E_{l}}{4 N_{0}}\right)^{-r M}}{Q_{l}} \text {. }
$$

It is easy to find that the optimal solution to (8) is

$$
E_{k}=E_{T} \frac{\sum_{i=1}^{K} L_{i} \cdot\left(\frac{1}{Q_{k}}\right)^{\frac{1}{r^{M}}}}{\sum_{i=1}^{K} L_{i}\left(\frac{1}{Q_{i}}\right)^{\frac{1}{r_{M}}}}
$$

which proves that large $Q_{k}$ leads to small $E_{k}$ and then higher $P e_{k}$.

\section{POWER OPTIMIZATION FOR BLOCK SPACE-TIME CODE}

Considering the decoding-complexity of the spacetime trellis coding, a simple yet powerful transmission scheme using two transmit antennas was developed in [5], and later was generalized to an arbitrary number of transmit antennas in [6]. These codes have a very simple maximum likelihood decoding algorithm based only on linear processing at the receiver, and are able to achieve the full diversity promised by the transmit and receive antennas. To deliver layer coded data using such codes, the power optimization can also be applied to further improve the performance. For 4-PSK modulation, the full diversity using $L$ transmit antennas and one receive antennas yields a BER

$P e(E)=\frac{1}{2}\left(1-\sqrt{\frac{E / N_{0}}{2+E / N_{0}}} \sum_{i=0}^{L-1}\left(\begin{array}{c}2 i \\ i\end{array}\right)\left\{2\left(2+E / N_{0}\right)\right\}^{-i}\right)$,

where $E / N_{0}$ is the SNR at each transmit antenna. Similarly to (4), the power distribution can be derived by using LaGrange multiplier.

\section{SIMULATION RESULT}

Subband coded image is a typical example of layer coded multimedia data. After subband decomposition, the subbands are vector quantized(VQ) and the indices are fixed length coded to achieve more robustness.
Each coded subband is recognized as a layer and the weight factor associated is computed. We compare the power optimized system with the conventional system with equal power. The system has multiple transmit antennas and one receive antennas. 4-PSK 8 states space-time code similar to [4] is employed. We assume flat Rayleigh fading with 40 and $180 \mathrm{~Hz}$ maximum Doppler frequency. Figure 3 plots the received PSNR vs. averaged channel SNR $\left(\frac{E_{T}}{N_{0}}\right)$ using subband coded image "Lena" at $0.5 \mathrm{bpp}$ using two transmitted antennas. As can be seen, the proposed algorithm achieves as much as 6dB PSNR improvement for low SNR such as $10 \mathrm{~dB}$ range. Image results at 16 and $18 \mathrm{~dB}$ SNR are shown in Figure 6 and 7. The performance using four transmit antennas and rate half space-time block code is illustrated at Figure 4 where as much as $4 \mathrm{~dB}$ PSNR improvement is achieved.

The proposed power optimized space-time code can be applied to transmit the integrated service, as to reduce the power consumption while maintaining the QoS requirement. We assume three services: video, speech and email, each with different data rate and QoS requirement as well as the $Q_{k}$, shown in Table 1. The BER performances are illustrated in Figure 5 . As can be seen, 29.5dB SNR can achieve the required $10^{-6}, 10^{-5}, 10^{-3}$ BER distribution. And $24 \mathrm{~dB}$ SNR yields $10^{-4}, 10^{-3}, 10^{-1}$.

Table 1. Data Rate and QoS for Various Services

\begin{tabular}{|c|c|c|c|}
\hline Service & Data Rate & BER & $Q_{k}$ \\
\hline \hline Video Conferencing & $200 \mathrm{~K}$ bits $/ \mathrm{s}$ & $10^{-6}$ & $10^{-3}$ \\
\hline Voice & $10 \mathrm{~K}$ bits $/ \mathrm{s}$ & $10^{-3}$ & 1 \\
\hline E-mail & $64 \mathrm{~K}$ bits $/ \mathrm{s}$ & $10^{-5}$ & $10^{-2}$ \\
\hline
\end{tabular}

\section{CONCLUSION}

We propose a power optimized space-time coding scheme for layer coded multimedia data transmission over wireless channels. Unequal error protection is achieved by allocating transmitted power according to the layer's perceptual importance. We discuss the power optimization for different space-time codes and for different performance requirement. Simulation results show 1-6dB PSNR improvement over equal power system for $10-22 \mathrm{~dB}$ average channel SNR.

\section{REFERENCES}

[1] A. Wittneben, "A New Bandwidth Efficient Transmit Antenna Modulation Diversity scheme for Linear Digital Modulation",Proc. of ICC'93, pp.16301634, June 1993.

[2] J.H.Winters, "The Diversity Gain of Transmit Diversity in Wireless Systems with Rayleigh Fading", 


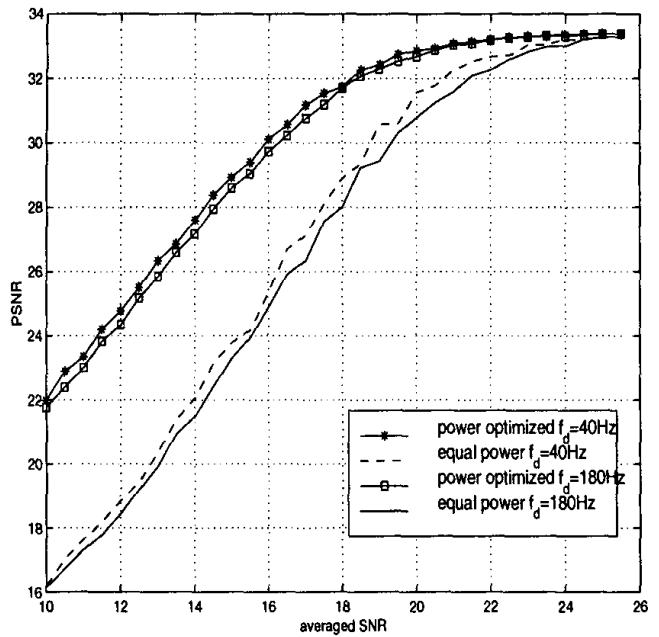

Figure 3: PSNR vs. average channel SNR comparison using two transmit antennas and space-time trellis code.

Proc. of ICC'94,pp.1121-1125, June 1994.

[3] G. W. Wornell and M.D. Troot, "Efficient Signal Processing Techniques for Exploiting Transmit Antenna Diversity on Fading Channels", IEEE Trans. on Signal Processing,pp. 191-205, Jan. 1997.

[4] V. Tarokh, N. Seshadri and A.R. Calderbank, "Space-Time Codes for High Data Rate Wireless Communication:Performance analysis and code construction", IEEE Trans. on Information Theory, pp.744-765, vol.44, No.2, March 1998.

[5] S.M. Alamouti, "A Simple Transmitter Diversity Scheme for Wireless Communications", IEEE J. Select. Areas Commun., vol.16, pp 1451-1458, Oct. 1998.

[6] V. Tarokh, H. Jafarkhani and A.R. Calderbank, "Space-Time Block Coding for Wireless Communications: Performance Results", IEEE J. Select. Areas Commun., pp.451-460, vol.17, No. 3, March 1999.

[7] H. Zheng and K.J.R. Liu, "Power and Rate Allocation Framework for Subband Image and Video Transmission", IEEE Trans. on Circuit and System for Video Technology, August 1999, to appear.

[8] H. Zheng and K.J.R. Liu, "Robust Image and Video Transmission over Spectrally Shaped Channels Using Multicarrier Modulation", IEEE Trans. on Multimedia, pp. 88-103, vol. 1, No. 1, March 1999.

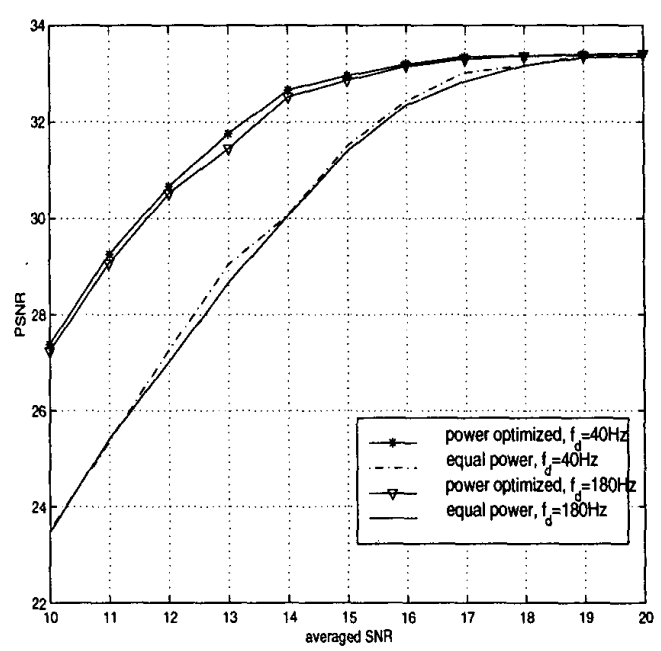

Figure 4: PSNR vs. average channel SNR comparison using four transmit antennas and space-time block code.

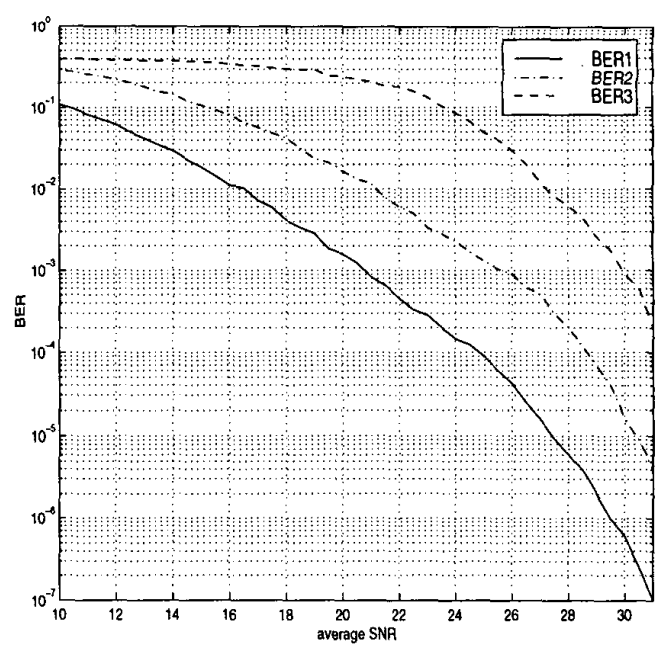

Figure 5: BER performance of integrated services. 


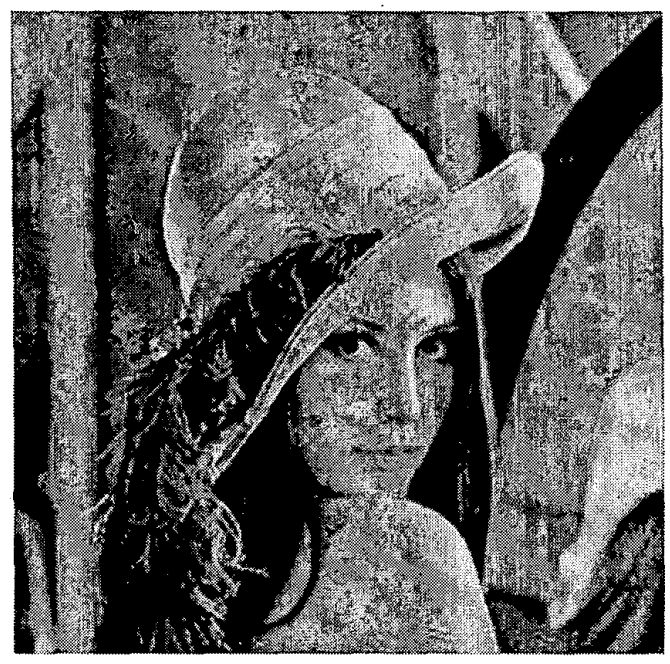

power optimized

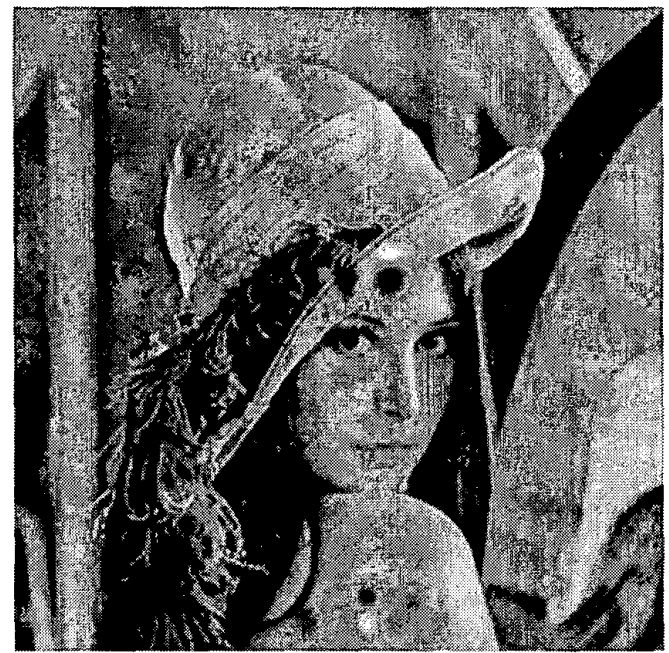

equal power

Figure 6: Received image comparison at $\mathrm{SNR}=18 \mathrm{~dB}$

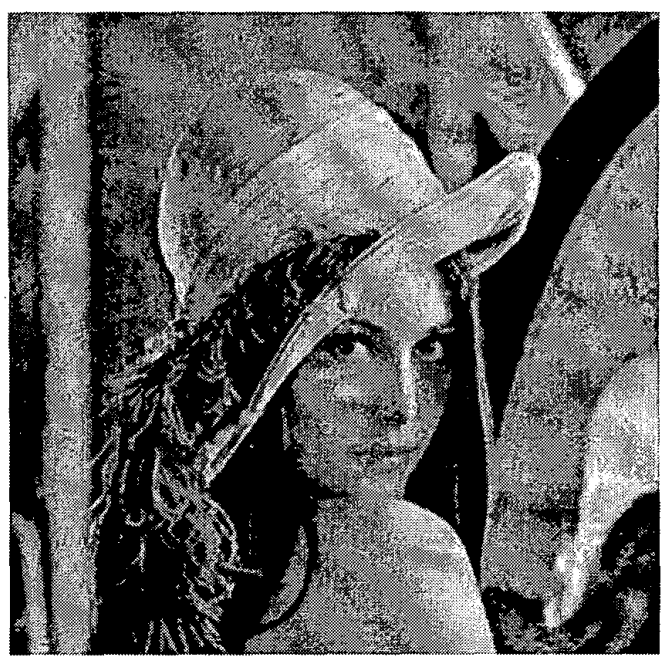

power optimized

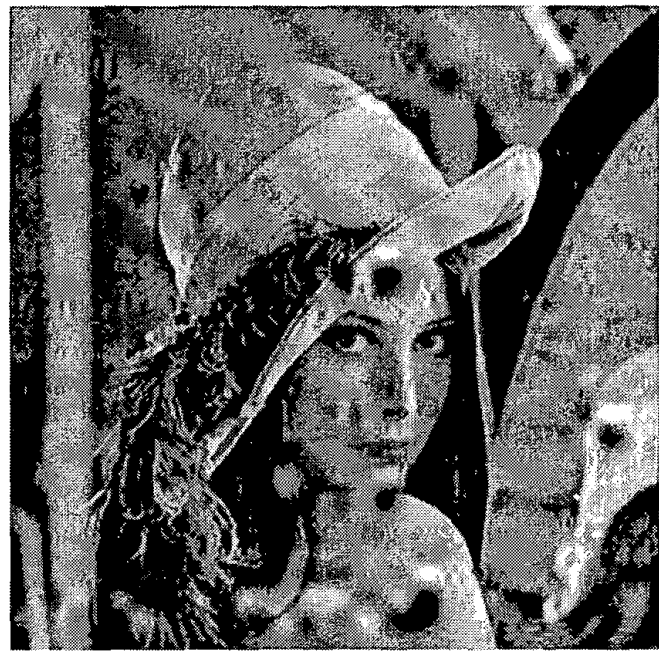

equal power

Figure 7: Received image comparison at $\mathrm{SNR}=16 \mathrm{~dB}$ 